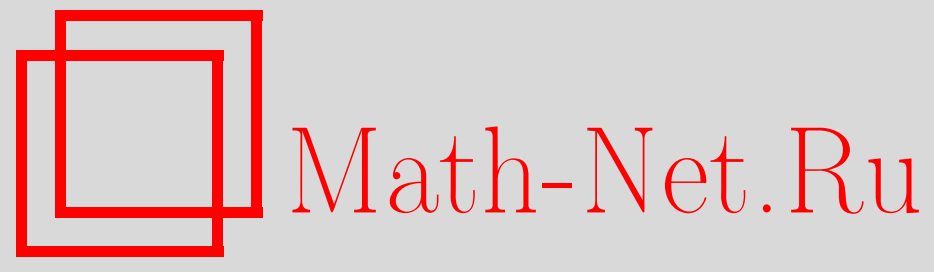

В. В. Лебедев, О равномерной сходимости рядов Фурье, Матем. заметки, 2012, том 91, выпуск 6, 946-949

DOI: https://doi.org/10.4213/mzm9165

Использование Общероссийского математического портала Math-Net.Ru подразумевает, что вы прочитали и согласны с пользовательским соглашением http://www . mathnet.ru/rus/agreement

Параметры загрузки:

IP: 54.162 .27 .143

26 апреля 2023 г., $15: 32: 21$

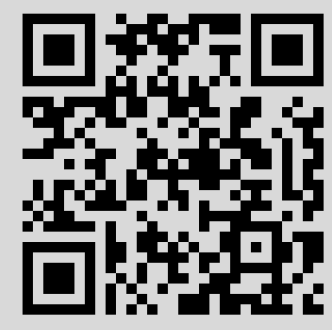




\section{О равномерной сходимости рядов Фурье}

\section{В. В. Лебедев}

Для произвольной интегрируемой функции $f$ на окружности $\mathbb{T}=\mathbb{R} / 2 \pi \mathbb{Z}$ (где $\mathbb{R}$ - вещественная прямая и $\mathbb{Z}$ - множество целых чисел) рассмотрим ее ряд Фурье

$$
f(t) \sim \sum_{k \in \mathbb{Z}} \widehat{f}(k) e^{i k t}, \quad \widehat{f}(k)=\frac{1}{2 \pi} \int_{\mathbb{T}} f(t) e^{-i k t} d t .
$$

Пусть $C(\mathbb{T})$ - пространство непрерывных функций $f$ на $\mathbb{T}$ с обычной нормой $\|f\|_{C(\mathbb{T})}=$ $\sup _{t \in \mathbb{T}}|f(t)|$. Пусть $U(\mathbb{T})$ - пространство всех функций $f \in C(\mathbb{T})$, ряд Фурье которых равномерно сходится, т.е. $\left\|S_{N}(f)-f\right\|_{C(\mathbb{T})} \rightarrow 0$ при $N \rightarrow \infty$, где $S_{N}(f)$ есть $N$-я частичная сумма ряда Фурье функции $f$ :

$$
S_{N}(f)(t)=\sum_{|k| \leqslant N} \widehat{f}(k) e^{i k t}
$$

Снабженное естественной нормой

$$
\|f\|_{U(\mathbb{T})}=\sup _{N}\left\|S_{N}(f)\right\|_{C(\mathbb{T})},
$$

пространство $U(\mathbb{T})$ является банаховым пространством.

Рассмотрим также пространство $A(\mathbb{T})$ функций $f \in C(\mathbb{T})$, имеющих абсолютно сходящийся ряд Фурье. Положим

$$
\|f\|_{A(\mathbb{T})}=\|\widehat{f}\|_{l^{1}}=\sum_{k \in \mathbb{Z}}|\widehat{f}(k)| .
$$

Пространство $A(\mathbb{T})$ банахово. Имеем $A(\mathbb{T}) \subset U(\mathbb{T})$ и $\|\cdot\|_{U(\mathbb{T})} \leqslant\|\cdot\|_{A(\mathbb{T})}$.

Пусть $\varphi$ - непрерывное отображение окружности $\mathbb{T}$ в себя, т.е. непрерывная функция $\varphi: \mathbb{R} \rightarrow \mathbb{R}$ такая, что $\varphi(t+2 \pi)=\varphi(t)(\bmod 2 \pi), t \in \mathbb{R}$. Согласно известной теореме Берлинга-Хелсона [1] (см. также [2], [3]), если $\left\|e^{i n \varphi}\right\|_{A(\mathbb{T})}=O(1), n \rightarrow \infty$, то функция $\varphi$ линейна. Вместе с тем известно, что существуют нетривиальные отображения $\varphi: \mathbb{T} \rightarrow \mathbb{T}$ такие, что $\left\|e^{i n \varphi}\right\|_{U(\mathbb{T})}=O(1)$. Обзор ряда результатов об отображениях окружности и пространствах $A(\mathbb{T}), U(\mathbb{T})$ имеется в работах [3], [4]. Более поздние результаты получены в [5].

Кахану принадлежит следующее утверждение [2; гл. IV]. Если нелинейное непрерывное отображение $\varphi: \mathbb{T} \rightarrow \mathbb{T}$ кусочно линейно (т.е. таково, что $[0,2 \pi]$ является конечным объединением интервалов, на каждом из которых функция $\varphi$ линейна), то $\left\|e^{i n \varphi}\right\|_{A(\mathbb{T})} \simeq \log |n|$. (Знак $\simeq$ означает, что при всех достаточно больших $n \in \mathbb{Z}$ отношение соответствующих величин заключено между двумя положительными константами.) Здесь мы получим аналог этого утверждения для пространства $U(\mathbb{T})$.

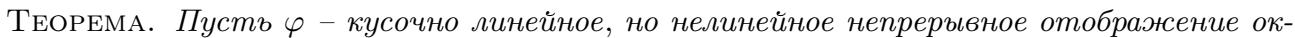
ружности $\mathbb{T}$ в себя. Тогда

$$
\left\|e^{i n \varphi}\right\|_{U(\mathbb{T})} \simeq \log |n|, \quad n \in \mathbb{Z} .
$$

Из этой теоремы, в частности, вытекает, что нетривиальные кусочно линейные замены переменной, вообще говоря, разрушают равномерную сходимость рядов Фурье. Более того, они не действуют из $A(\mathbb{T})$ в $U(\mathbb{T})$. В самом деле, предположив, что для любой функции $f \in A(\mathbb{T})$ суперпозиция $f \circ \varphi$ принадлежит $U(\mathbb{T})$, мы имели бы $\left\|e^{i n \varphi}\right\|_{U(\mathbb{T})}=O(1)$ (достаточно применить теорему о замкнутом графике к оператору $f \rightarrow f \circ \varphi)$.

Для доказательства теоремы надо лишь доказать $(\log |n|)$-оценку снизу; оценка сверху $\left\|e^{i n \varphi}\right\|_{U(\mathbb{T})}=O(\log |n|)$ следует из неравенства $\|\cdot\|_{U(\mathbb{T})} \leqslant\|\cdot\|_{A(\mathbb{T})}$ и указанного выше результата Кахана.

(C) В. В. ЛЕьедев, 2012 
Лемма. Пусть $m \in C(\mathbb{T})-$ функиия такая, что

$$
\|m\|_{*}=\sum_{n \in \mathbb{Z}}|\widehat{m}(n)| \log (|n|+2)<\infty .
$$

Тогда для любой функиии $f \in U(\mathbb{T})$ имеем $m f \in U(\mathbb{T})$ u

$$
\|m f\|_{U(\mathbb{T})} \leqslant c\|m\|_{*}\|f\|_{U(\mathbb{T})},
$$

где константа с $>0$ не зависит от $f$ u $\mathrm{m}$.

Доказательство. Пусть $f \in U(\mathbb{T})$. При $n \in \mathbb{Z}$ положим $e_{n}(t)=e^{i n t}$. Пусть $n>0$. Тогда

$$
S_{N}\left(e_{n} f\right)=e_{n} S_{N+n}(f)+e_{N} \widehat{f}(N-n)-e_{N+n} S_{n}\left(e_{-N} f\right) .
$$

Легко увидеть, что отсюда следует включение $e_{n} f \in U(\mathbb{T})$.

Для любой функции $g \in U(\mathbb{T})$ мы имеем $\|g\|_{C(\mathbb{T})} \leqslant\|g\|_{U(\mathbb{T})}$. Вместе с тем (как хорошо известно) для любой функции $g \in C(\mathbb{T})$ имеем

$$
\left\|S_{n}(g)\right\|_{C(\mathbb{T})} \leqslant c\|g\|_{C(\mathbb{T})} \log (n+2)
$$

c константой $c>0$, не зависящей от $n$ и $g$. Поэтому (1) дает

$$
\begin{aligned}
\left\|S_{N}\left(e_{n} f\right)\right\|_{C(\mathbb{T})} & \leqslant\left\|S_{N+n}(f)\right\|_{C(\mathbb{T})}+\|f\|_{C(\mathbb{T})}+\left\|S_{n}\left(e_{-N} f\right)\right\|_{C(\mathbb{T})} \\
& \leqslant\|f\|_{U(\mathbb{T})}+\|f\|_{C(\mathbb{T})}+c\|f\|_{C(\mathbb{T})} \log (n+2) \leqslant c_{1}\|f\|_{U(\mathbb{T})} \log (n+2) .
\end{aligned}
$$

Таким образом, $\left\|e_{n} f\right\|_{U(\mathbb{T})} \leqslant c_{1}\|f\|_{U(\mathbb{T})} \log (|n|+2)$. Такое же соотношение верно при $n<0$ (комплексное сопряжение не меняет норму функции в $U(\mathbb{T})$ ). Отсюда немедленно получаем утверждение леммы.

ДокАЗАТЕЛЬСТво теОРемЫ. Стандартным образом будем отождествлять функции на $\mathbb{T}$ и функции на отрезке $[-\pi, \pi]$. При $v \in \mathbb{R}$ определим функции $e_{v}$ на $[-\pi, \pi]$, полагая $e_{v}(t)=e^{i v t}$. Для произвольного интервала $I \subseteq[-\pi, \pi]$ пусть $1_{I}-$ его характеристическая функция: $1_{I}(t)=1$ при $t \in I, 1_{I}(t)=0$ при $t \in[-\pi, \pi] \backslash I$. При $0<\varepsilon<\pi$ пусть $\Delta_{\varepsilon}-$ “треугольная" функция, сосредоточенная на интервале $(-\varepsilon, \varepsilon)$, т.е. функция на $[-\pi, \pi]$, определенная соотношением $\Delta_{\varepsilon}(t)=\max (0,1-|t| / \varepsilon)$.

Пусть $t_{0}$ - такая точка, что функция $\varphi$ линейна в некоторой левой полуокрестности точки $t_{0}$ и линейна в некоторой правой полуокрестности точки $t_{0}$, но не линейна ни в какой ее окрестности. Заменяя (при необходимости) функцию $\varphi(t)$ на $\varphi\left(t+t_{0}\right)-\varphi\left(t_{0}\right)$, можем считать, что $t_{0}=0$ и $\varphi(0)=0$; таким образом, можем считать, что для некоторого $\varepsilon$, $0<\varepsilon<\pi$, имеем $\varphi(t)=\alpha t$ при $t \in(-\varepsilon, 0]$ и $\varphi(t)=\beta t$ при $t \in[0, \varepsilon)$, где $\alpha \neq \beta$.

Непосредственное вычисление дает при $k \neq n \alpha, n \beta$

$$
\widehat{\Delta_{\varepsilon} e^{i n \varphi}}(k)=\frac{1}{2 \pi i}\left(\frac{1}{n \alpha-k}-\frac{1}{n \beta-k}\right)-\frac{1}{i \varepsilon}\left(\frac{1}{n \alpha-k} \widehat{e_{n \alpha} 1_{(-\varepsilon, 0)}}(k)-\frac{1}{n \beta-k} \widehat{e_{n \beta} 1_{(0, \varepsilon)}}(k)\right) .
$$

При $\lambda \in \mathbb{R}$ пусть

$$
Q(\lambda)=\sum_{k \in \mathbb{Z}:|k-\lambda| \geqslant 1} \frac{1}{(k-\lambda)^{2}} .
$$

Легко проверить, что

$$
Q(\lambda) \leqslant 4, \quad \lambda \in \mathbb{R} .
$$

Покажем сначала, что при $n \in \mathbb{Z}, n \neq 0$, мы имеем

$$
\left\|\Delta_{\varepsilon} e^{i n \varphi}\right\|_{U(\mathbb{T})} \geqslant \frac{1}{2 \pi} \log |n|+c(\varphi),
$$

где $c(\varphi)$ не зависит от $n$.

Если $g \in U(\mathbb{T})$, то функция $g(-t)$ и функция $\overline{g(t)}$ (полученная комплексным сопряжением) принадлежат $U(\mathbb{T})$ и имеют ту же норму, что и $g$. Поэтому при доказательстве оценки (4) мы можем считать, что $\alpha>0$ и рассматривать лишь следующие три случая: 

1) $|\beta|>\alpha$
2) $\beta=-\alpha$;
3) $\beta=0$.

Мы можем также считать, что $n$ положительно и настолько велико, что $n \alpha \geqslant 2$. Всюду далее мы полагаем $N=[n \alpha]-1$, где $[x]$ означает целую часть числа $x$.

Случай 1). Имеем

$$
\begin{aligned}
\left|\sum_{|k| \leqslant N} \frac{1}{n \alpha-k}\right| & =\sum_{|k| \leqslant N} \frac{1}{n \alpha-k} \geqslant \sum_{|k| \leqslant N} \frac{1}{N+2-k} \\
& =\frac{1}{2}+\frac{1}{3}+\cdots+\frac{1}{2 N+2} \geqslant \log (N+1) \geqslant \log \frac{n \alpha}{2} .
\end{aligned}
$$

Вместе с тем, при $|k| \leqslant N$ имеем $|n \beta-k| \geqslant|n \beta|-|n \alpha|=n(|\beta|-\alpha)$, поэтому

$$
\left|\sum_{|k| \leqslant N} \frac{1}{n \beta-k}\right| \leqslant \frac{2 N+1}{n(|\beta|-\alpha)} \leqslant \frac{3 n \alpha}{n(|\beta|-\alpha)}=\frac{3 \alpha}{|\beta|-\alpha} .
$$

Заметим далее, что, пользуясь неравенством Коши и равенством Парсеваля, получаем (cM. (3))

$$
\left|\sum_{|k| \leqslant N} \frac{1}{n \alpha-k} \widehat{e_{n \alpha} 1_{(-\varepsilon, 0)}}(k)\right| \leqslant(Q(n \alpha))^{1 / 2}\left\|e_{n \alpha} 1_{(-\varepsilon, 0)}\right\|_{L^{2}(\mathbb{T})} \leqslant 2 \varepsilon^{1 / 2},
$$

и аналогично

$$
\left|\sum_{|k| \leqslant N} \frac{1}{n \beta-k} \widehat{e_{n \beta} 1_{(0, \varepsilon)}}(k)\right| \leqslant(Q(n \beta))^{1 / 2}\left\|e_{n \beta} 1_{(0, \varepsilon)}\right\|_{L^{2}(\mathbb{T})} \leqslant 2 \varepsilon^{1 / 2} .
$$

Вместе соотношения (5)-(8) влекут (см. (2))

$$
\left|S_{N}\left(\Delta_{\varepsilon} e^{i n \varphi}\right)(0)\right|=\left|\sum_{|k| \leqslant N} \widehat{\Delta_{\varepsilon} e^{i n \varphi}}(k)\right| \geqslant \frac{1}{2 \pi}\left(\log \frac{n \alpha}{2}-\frac{3 \alpha}{|\beta|-\alpha}\right)-4 \varepsilon^{-1 / 2},
$$

и мы получаем (4).

Случай 2). Имеем

$$
\left|\sum_{|k| \leqslant N}\left(\frac{1}{n \alpha-k}-\frac{1}{n \beta-k}\right)\right|=\left|\sum_{|k| \leqslant N}\left(\frac{1}{n \alpha-k}+\frac{1}{n \alpha+k}\right)\right|=\left|2 \sum_{|k| \leqslant N} \frac{1}{n \alpha-k}\right| \geqslant \log \frac{n \alpha}{2} .
$$

Вместе с оценками (7), (8), которые верны в случае 2), эта оценка дает

$$
\left|S_{N}\left(\Delta_{\varepsilon} e^{i n \varphi}\right)(0)\right| \geqslant \frac{1}{2 \pi} \log \frac{n \alpha}{2}-4 \varepsilon^{-1 / 2},
$$

и мы опять получаем (4).

Случай 3). Имеем

$$
\left|\sum_{1 \leqslant|k| \leqslant N}\left(\frac{1}{n \alpha-k}-\frac{1}{n \beta-k}\right)\right|=\left|\sum_{1 \leqslant|k| \leqslant N}\left(\frac{1}{n \alpha-k}-\frac{1}{-k}\right)\right|=\left|\sum_{1 \leqslant|k| \leqslant N} \frac{1}{n \alpha-k}\right| \geqslant \log \frac{n \alpha}{2}-1 .
$$

Заметим, что оценки (7), (8) верны в случае 3$)$, если промежуток $|k| \leqslant N$ в суммах заменить на $1 \leqslant|k| \leqslant N$. Таким образом, видим, что

$$
\left|\sum_{1 \leqslant|k| \leqslant N} \widehat{\Delta_{\varepsilon} e^{i n \varphi}}(k)\right| \geqslant \frac{1}{2 \pi}\left(\log \frac{n \alpha}{2}-1\right)-4 \varepsilon^{-1 / 2},
$$


и, поскольку $\left|\widehat{\Delta_{\varepsilon} e^{i n \varphi}}(0)\right| \leqslant 1$, получаем

$$
\left|S_{N}\left(\Delta_{\varepsilon} e^{i n \varphi}\right)(0)\right| \geqslant \frac{1}{2 \pi}\left(\log \frac{n \alpha}{2}-1\right)-4 \varepsilon^{-1 / 2}-1 .
$$

Оценка (4) доказана.

Заметим теперь, что $\widehat{\Delta_{\varepsilon}}(k)=O\left(1 /|k|^{2}\right)$ при $|k| \rightarrow \infty$, поэтому

$$
\left\|\Delta_{\varepsilon}\right\|_{*}=\sum_{k \in \mathbb{Z}}\left|\widehat{\Delta_{\varepsilon}}(k)\right| \log (|k|+2)=M(\varepsilon)<\infty,
$$

и из (4), пользуясь леммой, получаем

$$
c(\varphi)+\frac{1}{2 \pi} \log |n| \leqslant\left\|\Delta_{\varepsilon} e^{i n \varphi}\right\|_{U(\mathbb{T})} \leqslant c M(\varepsilon)\left\|e^{i n \varphi}\right\|_{U(\mathbb{T})} .
$$

Теорема доказана.

ЗАмЕчАниЕ. Было бы интересно описать поточечные мультипликаторы пространства $U(\mathbb{T})$, т.е. такие непрерывные функции $m$ на $\mathbb{T}$, что $m f \in U(\mathbb{T})$ для любой функции $f \in$ $U(\mathbb{T})$. Согласно полученной выше лемме, условие

$$
\sum_{k \in \mathbb{Z}}|\widehat{m}(k)| \log (|k|+2)<\infty
$$

является достаточным для того, чтобы функция $m$ являлась мультипликатором. Автору не известноб является ли это условие необходимым. Указанное условие нельзя заменить более слабым условием $m \in A(\mathbb{T})$ (см. [2; гл. I, §6]).

\section{СПИСОК ЦИТИРОВАННОЙ ЛИТЕРАТУРЫ}

[1] A. Beurling, H. Helson, Math. Scand., 1 (1953), 120-126. [2] Ж.-П. Кахан, Абсолютно сходящиеся ряды Фуръе, Мир, М., 1976. [3] J.-P. Kahane, Topics in Modern Harmonic Analysis, Vol. II, Ist. Naz. Alta Mat. Francesco Severi, Roma, 1983, 955-990. [4] А. М. Олевский, УМН, 40:3 (1985), 157-193. [5] В. В. Лебедев, Матем. сб., 201:12 (2010), 103-130.

В. В. Лебедев

Московский государственный институт электроники и математики (технический университет)

E-mail: lebedevhome@gmail.com
Поступило 26.05.2011

Исправленный вариант

19.01.2012 